\title{
Primitive Permutation Groups and Strongly Factorizable Transformation Semigroups
}

\author{
João Araújo*†, Wolfram Bentz; and Peter J. Cameron ${ }^{\S}$
}

\begin{abstract}
Let $\Omega$ be a finite set and $T(\Omega)$ be the full transformation monoid on $\Omega$. The rank of a transformation $t \in T(\Omega)$ is the natural number $|\Omega t|$. Given $A \subseteq T(\Omega)$, denote by $\langle A\rangle$ the semigroup generated by $A$. Let $k$ be a fixed natural number such that $2 \leq k \leq|\Omega|$. In the first part of this paper we (almost) classify the permutation groups $G$ on $\Omega$ such that for all rank $k$ transformations $t \in T(\Omega)$, every element in $S_{t}:=\langle G, t\rangle$ can be written as a product $e g$, where $e^{2}=e \in S_{t}$ and $g \in G$. In the second part we prove, among other results, that if $S \leq T(\Omega)$ and $G$ is the normalizer of $S$ in the symmetric group on $\Omega$, then the semigroup $S G$ is regular if and only if $S$ is regular. (Recall that a semigroup $S$ is regular if for all $s \in S$ there exists $s^{\prime} \in S$ such that $s=s s^{\prime} s$.) The paper ends with a list of problems.
\end{abstract}

\footnotetext{
*Departamento de Matemática and CMA, Faculdade de Ciências e Tecnologia (FCT) Universidade Nova de Lisboa (UNL), 2829-516 Caparica, Portugal; jj.araujo@fct.unl.pt

${ }^{\dagger}$ CEMAT-Ciências, Faculdade de Ciências, Universidade de Lisboa 1749-016, Lisboa, Portugal; jjaraujo@fc.ul.pt

${ }^{\ddagger}$ Department of Physics \& Mathematics, University of Hull, Kingston upon Hull, HU6 7RX, UK; W.Bentz@hull.ac.uk

${ }^{\S}$ School of Mathematics and Statistics, University of St Andrews, North Haugh, St Andrews, Fife KY16 9SS, UK; pjc20@st-andrews.ac.uk
} 


\section{Introduction}

A semigroup $S$ with set of idempotents $E$ and group of units $G$ is said to be strongly factorizable if $S=E G$. Denote by $S_{n}$ the symmetric group on

a set $\Omega$ of cardinality $n$ and denote by $T_{n}$ the full transformation semigroup on the same set. It is clear that $S_{n}$ is the group of units of $T_{n}$. Given $t \in T_{n}$ the rank of $t$ is the cardinality of the set $\Omega t$.

The first goal of this paper is to prove the following sequence of theorems. We note that, for $1<k<n-1$, the $k$-transitive or $k$-homogeneous groups are explicitly known.

Theorem 1.1 Let $n \geq 2$, and $G \leq S_{n}$ be a group. The following are equivalent:

(a) for all rank 2 transformations $t \in T_{n}$ the semigroup $\langle G, t\rangle$ is strongly factorizable;

(b) $G$ is primitive.

Theorem 1.2 Let $G \leq S_{n}$ and let $k$ be a fixed natural number such that $6 \leq k \leq n$. The following are equivalent:

(a) for all rank $k$ transformations $t \in T_{n}$ the semigroup $\langle G, t\rangle$ is strongly factorizable;

(b) $G=S_{n}$ or $G=A_{n}$ (with $n \neq k$ ) in their natural action on $n$ points.

Theorem 1.3 Let $n \geq 5$ and $G \leq S_{n}$. The following are equivalent:

(a) for all rank 5 transformations $t \in T_{n}$ the semigroup $\langle G, t\rangle$ is strongly factorizable;

(b) $G$ is 5-transitive or $G=A_{6}(n=6)$.

Theorem 1.4 Let $n \geq 4$ and $G \leq S_{n}$. If $G$ is 4-transitive, or $G$ is one of $A_{5}$ $(n=5)$, or $M_{11}(n=12)$, then the semigroup $\langle G, t\rangle$ is strongly factorizable, for all rank 4 transformations $t \in T_{n}$.

Any other group satisfying this property contains $\operatorname{PSL}\left(2,2^{p}\right)\left(n=2^{p}+1\right)$, where $2^{p}-1$ is a (Mersenne) prime.

Theorem 1.5 Let $n \geq 3$ and $G \leq S_{n}$. If $G$ satisfies one of the following properties: 
(a) $G$ is 3-transitive;

(b) $\operatorname{PSL}(2, q) \leq G \leq \operatorname{P\Sigma L}(2, q)$ where $q$ is an odd prime.

(c) $G=\operatorname{Sp}(2 d, 2), d \geq 3$, in either of its 2-transitive representations ( $n=$ $\left.2^{2 d-1} \pm 2^{d-1}\right)$

(d) $G=2^{d}: \operatorname{Sp}(d, 2), d \geq 4$ and even $\left(n=2^{d}\right)$;

(e) $G$ is one of $A_{4}(n=4), \operatorname{PSL}(2,11)(n=11), 2^{4}: A_{6}(n=16)$, $2^{6}: G_{2}(2)$ or its subgroup of index $2(n=64)$, Higman-Sims $(n=176)$, $\mathrm{Co}_{3}(n=276)$;

then the semigroup $\langle G, t\rangle$ is strongly factorizable, for all rank 3 transformations $t \in T_{n}$.

Any other group satisfying the previous property must satisfy $\operatorname{AGL}\left(1,2^{p}\right) \leq$ $G \leq \mathrm{A} \Gamma \mathrm{L}\left(1,2^{p}\right)$ with $p$ and $2^{p}-1$ prime.

These results required the repeated use of the classification of finite simple groups. This completes the part of the paper dealing with semigroups $S$ in which $S=E G$.

In the second part we turn to semigroups of the form $S G$, where $S \leq T_{n}$ and $G$ is the normalizer of $S$ in $S_{n}$. The general goal is to decide if some properties of $S G$ carry to $S$. The main theorem is the following.

Theorem 1.6 Let $S \leq T_{n}$ be a transformation monoid and $G$ be the normalizer of $S$ in the symmetric group $S_{n}$. Then $S G$ is regular if and only if $S$ is regular.

As often happens in semigroup theory, the proof is short but tricky.

Finally we prove the following result that generalizes a well known theorem in groups.

Theorem 1.7 Let $S$ be a finite semigroup, and $n$ a positive integer. Then every element of $S$ has an $n$-th root if and only if every element in $S$ belongs to a subgroup of order coprime to $n$.

A semigroup $S$ is said to be factorizable if there exist two sets $A, B \subset S$ such that $S=A B$. Factorizable semigroups were prompted by the study of extensions (direct products, Zappa-Szép and Catino extensions [11]), but the 
study went far beyond that initial motivation leading to many papers in very different contexts (structural semigroup theory, topological semigroups, presentations, automata and languages, combinatorial semigroup theory, Morita equivalences, etc.). If $S=E G$, where $E$ is its set of idempotents and $G$ is its group of units, then $S$ is said to be strongly factorizable. The class of strongly factorizable semigroups contains the two most studied transformation semigroups: the full transformation monoid on a finite set and the monoid of endomorphisms of a finite-dimensional vector space. As, by an argument similar to the one used to prove Cayley's Theorem in groups, every finite semigroup embeds in some finite $T_{n}$, it follows that every finite semigroup is a subsemigroup of a strongly factorizable semigroup. Recall that a semigroup $S$ is said to be regular if for all $a \in S$ there exists $b \in S$ (called an inverse of $a$ ) such that $a=a b a$ and $b=b a b$. If every element in a regular semigroup has only one inverse, then the semigroup is said to be inverse. Not every strongly factorizable semigroup is inverse (consider a non-commutative semigroup of idempotents with identity), but they are all regular semigroups. In fact, if $e g \in E G$ then $e g=(e g)\left(g^{-1} e\right)(e g)$ and $g^{-1} e=\left(g^{-1} e\right)(e g)\left(g^{-1} e\right)$.

In [16] the related problem of describing the structure of the subsemigroup $\langle E, G\rangle$ has been considered in the context of infinite semigroups, where variants using left and right units arise as well. Finally we note that in the context of inverse semigroups (where strongly factorizable semigroups are simply called factorizable) this topic is an entire body of knowledge on its own. (For details we suggest the lovely survey paper [12] and the references therein.) In the context of groups, it is usually assumed that at least one of $A$ and $B$ is a subgroup; many classifications are known, notably that of Liebeck, Praeger and Saxl [22,23], who found all factorizations of almost simple groups where both factors are subgroups.

Throughout the twentieth century there was a doctrine which stated that a problem in semigroups was considered solved when reduced to a question in groups. This dramatically changed in this century when it was realized that it would be much more productive for both sides to keep an ongoing conversation. One of the driving forces of this conversation has been the following general problem, whose study has led to significant research on permutation groups:

Classify the pairs $(G, a)$, where $a$ is a map on a finite set $\Omega$ and $G$ is a group of permutations of $\Omega$, such that the semigroup $\langle G, a\rangle$ generated by $a$ and $G$ has a given property $P$. 
A very important class of groups that falls under this general scheme is that of synchronizing groups, groups of permutations on a set that together with any non-invertible map on the same set generate a constant (see $[2,6$, $8,10,13,14,25])$. These groups are very interesting from a group theoretic point of view and are linked to the Černý conjecture, a longstanding open problem in automata theory.

Three other sample instances of the general question are the following:

(a) Let $A \subseteq T_{n}$; classify the permutation groups $G \leq S_{n}$ such that $\langle G, a\rangle$ is regular for all $a \in A$. For many different sets $A$, this problem has been considered in $[3-5,7,19-21,24]$, among others.

(b) Classify the permutation groups $G \leq S_{n}$ such that for all $a \in T_{n} \backslash S_{n}$ the equality $\langle G, a\rangle \backslash G=\left\langle S_{n}, a\right\rangle \backslash S_{n}$ holds. This problem was solved in [1].

(c) Classify the permutation groups $G \leq S_{n}$ such that for all $a \in T_{n} \backslash S_{n}$ we have $\langle G, a\rangle \backslash S_{n}=\left\langle g^{-1} a g \mid g \in G\right\rangle$. This classification [9] answered an old problem.

We saw above the importance of strongly factorizable semigroups and the multitude of contexts in which they appear. The first goal of this paper is to continue the trend described above and classify the permutation groups that together with any transformation of a given rank $k$ generate a strongly factorizable semigroup.

The second part of the paper deals with the following problem proposed by the third author. Observe that in the founding paper of factorizable semigroups [26] the goal was to check when some given properties of $A$ and $B$ carry to the factorizable oversemigroup $S:=A B$. Here we go in the converse direction: given $T=S G$, where $S \leq T_{n}$ and $G$ is the normalizer of $S$ in $S_{n}$, find semigroup properties that carry from $S G$ to $S$. This looks a sensible question since in $S G$ we can take advantage of the group theory machinery and hence checking a property might be easier in $S G$ than in $S$.

The main result of this part of the paper says that regularity carries from $S G$ to $S$.

We now summarise the contents of the paper. In Section 2 we classify permutation groups with what we call the ordered $k$-ut property. This is the cornerstone of our classification results. Section 3 connects the group theory results of the previous section with the theorems on factorizable semigroups. 
Section 4 deals with the semigroups $S G$, where $S \leq T_{n}$ and $G$ is its normalizer in $S_{n}$. The paper finishes with a list of open problems.

\section{The ordered $k$-ut property}

A permutation group $G$ on $\Omega$ is said to have the $k$-universal transversal property (or $k$-ut for short) if, given any $k$-subset $A$ and $k$-partition $P$ of $\Omega$, there exists $g \in G$ such that $A g$ is a transversal to $P$. These groups were studied in connection with permutation groups $G$ such that $\langle G, a\rangle$ is regular for all maps $a$ of rank $k$. The groups satisfying the $k$-ut property for $3 \leq k \leq n / 2$ were partly classified in [5] (small corrections to the case of 3-ut where made in [2]).

A permutation group $G$ on $\Omega$ is said to have the ordered $k$-ut property if, given any ordered $k$-subset $A=\left(a_{1}, \ldots, a_{k}\right)$ and ordered $k$-partition $\pi=$ $\left(P_{1}, \ldots, P_{k}\right)$ of $\Omega$, there exists $g \in G$ such that $a_{i} g \in P_{i}$ for $i=1, \ldots, k$.

Our goal is to classify the groups possessing ordered $k$-ut. Clearly, ordered $k$-ut implies the usual $k$-ut, so we only need look among permutation groups with $k$-ut.

\subsection{Permutation group properties}

A permutation group is $k$-primitive if it is $k$-transitive and the stabiliser of $k-1$ points is primitive on the remaining points.

A permutation group is generously $k$-transitive if, given any $(k+1)$-set $M \subseteq \Omega$, the group induced on $M$ by its setwise stabiliser is the symmetric group of degree $k+1$. It is straightforward to prove that a generously $k$ transitive group is indeed $k$-transitive.

The next result summarises the relationship between these concepts and the ordered $k$-ut property.

Proposition $2.1 \quad$ (a) A k-transitive group has the ordered $k$-ut property.

(b) For $k \geq 2$, ordered $k$-ut implies ordered $(k-1)$-ut.

(c) A permutation group $G$ which has $k$-ut and is generously $(k-1)$ transitive has ordered $k$-ut.

(d) A permutation group $G$ with the ordered $k$-ut property is $(k-1)$ transitive. 
(e) A permutation group $G$ with the ordered $k$-ut property is $(k-1)$ primitive.

(f) For $k \geq 2$, if $G$ has ordered $k$-ut, its point stabiliser has ordered $(k-1)$ ut.

Proof (a), (b) and (f) are straightforward.

(c) Given a $k$-set $A$ and a $k$-partition $\pi$, there is an element of $G$ mapping $A$ to a transversal of $\pi$; premultiplying this element by an element in the setwise stabiliser of $A$ shows that we can map elements of $A$ to parts of $\pi$ in any order.

(d) Let $\left(a_{1}, \ldots, a_{k-1}\right)$ and $\left(b_{1}, \ldots, b_{k-1}\right)$ be two ordered $(k-1)$-tuples of distinct points of $\Omega$. If $x$ is any point different from $a_{1}, \ldots, a_{k-1}$, then a permutation mapping $a_{1}, \ldots, a_{k-1}, x$ to a transversal of the partition $\left\{b_{1}\right\}, \ldots$, $\left\{b_{k-1}\right\}, \Omega \backslash\left\{b_{1}, \ldots, b_{k-1}\right\}$ maps the first $(k-1)$-tuple to the second.

(e) Suppose that $G$ is not $(k-1)$-primitive; let $B$ be a non-trivial block of imprimitivity for the stabiliser of distinct $a_{1}, \ldots, a_{k-2} \in \Omega$. Let $A$ be a subset consisting of $a_{1}, \ldots, a_{k-2}$ and two points $b_{1}, b_{2}$ of $B$, and $P$ the partition into $\left\{a_{1}\right\}, \ldots,\left\{a_{k-2}\right\}, B$, and the rest of $\Omega$. Any permutation mapping $a_{i}$ to $a_{i}$ for $i=1, \ldots, k-2$, maps $b_{1}, b_{2}$ either both into $B$ or outside of $B$. Hence $G$ does not have the ordered $k$-ut property.

Proposition 2.2 A permutation group $G$ has the ordered 2-ut property if and only if it is primitive.

Proof Ordered 2-ut implies primitivity, by (e) above. Conversely, suppose $G$ is primitive. Then all orbital digraphs for $G$ are connected, and hence (since $G$ is transitive) strongly connected. Now let $A=\left\{a_{1}, a_{2}\right\}$ be a 2-set and $\pi=\left\{P_{1}, P_{2}\right\}$ a 2-partition. Since the orbital graph with edge set $\left(a_{1}, a_{2}\right)^{G}$ is strongly connected, there is an edge with initial vertex in $P_{1}$ and terminal vertex in $P_{2}$; the element of $G$ mapping $\left(a_{1}, a_{2}\right)$ to this edge witnesses ordered $k$-ut.

The next proposition gives sufficient conditions for generous $k$-transitivity.

Proposition 2.3 (a) Suppose that $G$ is $k$-transitive, and every orbital of the $(k-1)$-point stabiliser is self-paired. Then $G$ is generously $k$ transitive. 
(b) Suppose that $G$ is $k$-transitive, and the non-trivial orbits of the stabiliser of $k$ points all have different sizes. Then $G$ is generously $k$-transitive.

Proof (a) Take any $k+1$ points $a_{1}, \ldots, a_{k+1}$. By assumption, $G$ has an element fixing $a_{1}, \ldots, a_{k-1}$ and interchanging $a_{k}$ with $a_{k+1}$. Since the numbering of the points is arbitrary, the setwise stabiliser of the set of $k+1$ points induces every possible transposition on it. The transpositions generate the symmetric group.

(b) This follows immediately from (a), since paired orbits have the same sizes.

\subsection{The classification of the groups with the ordered $k$-ut property}

Trivially, $G \leq S_{n}$ has the ordered $n$-ut property if and only if $G=S_{n}$. Any permutation group has the ordered 1-ut property and by Proposition 2.2, ordered 2 -ut is equivalent to primitivity.

Ordered $k$-ut clearly implies $k$-ut, and hence (by Proposition 2.1(b)), $k^{\prime}$ ut for all $1 \leq k^{\prime} \leq k$. Hence it remains to consider the groups arising in the classification of groups with $k$-ut from $[2,5]$ (given below). If $n$ is not much larger than $k$, there are often sporadic cases to consider. Below, we will deal with these cases by specialized arguments.

Proposition 2.4 Let $n \geq 6, G \leq S_{n}$, then $G$ has the ordered $k$-ut property for some $6 \leq k \leq n$, if and only if $G=S_{n}$ or $G=A_{n}$ (with $n \neq k$ ) in their natural action on $n$ points.

Proof By [5, Theorem 1.4], for $n \geq 11$, the only groups with 6-ut are $A_{n}$ and $S_{n}$, hence no other group has ordered 6-ut, and so it does not have ordered $k$-ut either. For $n \leq 10$, the listed groups are the only ones that are $(k-1)$-transitive.

Conversely, it is easy to check that the listed values of $A_{n}$ and $S_{n}$ have the ordered $k$-ut property, for $6 \leq k \leq n$.

Proposition 2.5 Let $n \geq 5, G \leq S_{n}$, then $G$ has the ordered 5-ut property if and only if it is 5-transitive or $A_{6}(n=6)$.

Proof For $n \geq 11$, a group with 5-ut is 5-homogeneous or $\operatorname{P\Gamma L}(2,32)$ (with degree 33) [5, Theorem 1.5]. The 5-homogeneous groups (with $n \geq 10$ ) are 
5-transitive and have ordered 5-ut, while $\operatorname{P\Gamma L}(2,32)$ is not 4-transitive so does not have ordered 5-ut.

For $n \leq 10, A_{6}(n=6)$, which clearly satisfies ordered 5-ut, is the only group that is 4 -transitive, but not 5-transitive.

Proposition 2.6 Let $n \geq 4, G \leq S_{n}$, then $G$ has the ordered 4-ut property if it is 4-transitive, $A_{5}(n=5)$, or $M_{11}(n=12)$. If there are any other groups with ordered 4 -ut, they contain $\operatorname{PSL}\left(2,2^{p}\right)\left(n=2^{p}+1\right)$, where $2^{p}-1$ is a (Mersenne) prime.

Proof By [5, Theorems 1.3, 1.6] for $n \geq 8$, a group with 4-ut is 4-homogeneous or $M_{11}(n=12)$, or possibly almost simple with socle $\operatorname{PSL}(2, q)$ where $q$ is prime or $q=2^{p}$ for some prime $p$ (with $n=q+1$ ). The 4-homogeneous groups with $n \geq 8$ are 4-transitive except for $\operatorname{PSL}(2,8)$, $\operatorname{P\Gamma L}(2,8)(n=9)$, and $\operatorname{P\Gamma L}(2,32)(n=33)$.

For $4 \leq n \leq 7$, the only 3 -, but not 4 -transitive groups are $A_{5}(n=5)$ and PGL $(2,5)(n=6)$.

The 4-transitive groups have ordered 4-ut, and the Mathieu group $M_{11}$ $(n=12)$ is generously 3 -transitive (the orbit lengths for the 3-point stabiliser are 3 and 6 ), and thus also has ordered 4-ut. Except in the case where $q-1$ is a Mersenne prime, almost simple groups with socle $\operatorname{PSL}(2, q)$ with $q \geq 5$ are not 3-primitive, since the largest such group $\operatorname{P\Gamma L}(2, q)$ has the property that the stabiliser of two points has a normal cyclic subgroup of composite order $q-1$, and a subgroup of a non-3-primitive group is non-3-primitive.

Finally, $A_{5}$ clearly has ordered 4-ut.

We remark that computation shows that the groups $\operatorname{PSL}(2,8), \operatorname{P\Gamma L}(2,8)$ $(n=9)$, and $\mathrm{P} \Gamma \mathrm{L}(2,32)(n=33)$ satisfy ordered 4-ut.

Before we consider the case $k=3$, we will give an updated list of the status of the 3-ut property. The following theorem combines results from [5] with the corrections from [2].

Proposition 2.7 Let $n \geq 3, G \leq S_{n}$, then $G$ has the 3-ut property if it satisfies one of the following properties:

(a) $G$ is 3-homogeneous;

(b) $\operatorname{PSL}(2, q) \leq G \leq \operatorname{P\Sigma L}(2, q)$ where $q \equiv 1 \bmod 4(n=q+1)$; 
(c) $G=\operatorname{Sp}(2 d, 2), d \geq 3$, in either of its 2-transitive representations ( $n=$ $\left.2^{2 d-1} \pm 2^{d-1}\right)$;

(d) $G=2^{d}: \operatorname{Sp}(d, 2), d \geq 4$ and even $\left(n=2^{d}\right)$;

(e) $G$ is one of $C_{5}, D(2 * 5)(n=5), \operatorname{AGL}(1,7),(n=7), \operatorname{PSL}(2,11)$ $(n=11), 2^{4}: A_{6}(n=16), 2^{6}: G_{2}(2)$ or its subgroup of index 2 $(n=64), \mathrm{Sz}(8), \mathrm{Sz}(8): 3(n=65)$, Higman-Sims $(n=176), \mathrm{Co}_{3}$ $(n=276)$;

If there are any other groups with 3-ut, they are one of the following:

(f) Suzuki groups $\mathrm{Sz}(q)$ with $q \geq 32$, potentially extended by field automorphisms $\left(n=q^{2}+1\right)$;

(g) $\operatorname{AGL}(1, q) \leq G \leq \mathrm{A} \Gamma \mathrm{L}(1, q)$, where $q$ is either prime with $q \equiv 11$ mod 12 , or $q=2^{p}$ with $p$ prime, and for all $c \in \mathrm{GF}(q) \backslash\{0,1\},|\langle-1, c, c-1\rangle|=$ $q-1(n=q)$;

(h) subgroups of index 2 in $\mathrm{AGL}(1, q)$, with $q \equiv 11 \bmod 12$ and prime, and for all $c \in \mathrm{GF}(q) \backslash\{0,1\},|\langle-1, c, c-1\rangle|=q-1(n=q)$.

With this result, we can prove that the groups with ordered 3-ut are just those listed in Theorem 1.5.

Proposition 2.8 Let $n \geq 3, G \leq S_{n}$. If $G$ satisfies one of the following properties:

(a) $G$ is 3-transitive;

(b) $\operatorname{PSL}(2, q) \leq G \leq \operatorname{P} \Sigma \mathrm{L}(2, q)$ where $q$ is an odd prime power.

(c) $G=\operatorname{Sp}(2 d, 2), d \geq 3$, in either of its 2-transitive representations $(n=$ $\left.2^{2 d-1} \pm 2^{d-1}\right)$

(d) $G=2^{d}: \operatorname{Sp}(d, 2), d \geq 4$ and even $\left(n=2^{d}\right)$;

(e) $G$ is one of $A_{4}(n=4), \operatorname{PSL}(2,11)(n=11), 2^{4}: A_{6}(n=16)$, $2^{6}: G_{2}(2)$ or its subgroup of index $2(n=64)$, Higman-Sims $(n=176)$, $\mathrm{Co}_{3}(n=276)$; 
then it satisfies ordered 3-ut.

Any other group satisfying ordered 3-ut must satisfy $\operatorname{AGL}\left(1,2^{p}\right) \leq G \leq$ $\mathrm{A \Gamma L}\left(1,2^{p}\right)\left(n=2^{p}\right)$ with $p$ and $2^{p}-1$ prime.

Proof According to Proposition 2.7, groups with 3-ut are one of the five types in the proposition, or potentially one of the three additional types listed.

If $G$ is 3-transitive, then it has the ordered 3-ut property.

Suppose that $G$ is 3 -homogeneous but not 3-transitive. These groups were classified by Kantor [18]. If $3 \leq n \leq 5$, then $G$ is $A_{4}(n=4)$, which clearly has ordered 3 -ut, $\operatorname{AGL}(1,5)(n=5)$, which we will exclude below, or not 2-transitive and hence does not have ordered 3-ut.

If $n \geq 6$, then $G$ is $\operatorname{AGL}(1,8), \operatorname{A\Gamma L}(1,8)(n=8)$ or $\operatorname{A\Gamma L}(1,32)(n=32)$, or $G$ contains $\operatorname{PSL}(2, q)$ for $q \equiv 3(\bmod 4)(n=q+1)$. The affine groups are included in our undecided cases, so assume $G$ contains such a $\operatorname{PSL}(2, q)$.

Let $\left\{a_{1}, a_{2}, a_{3}\right\}$ be an ordered 3 -set and $\left\{P_{1}, P_{2}, P_{3}\right\}$ an ordered 3-partition of the underlying set $\Omega$. Without loss of generality, we may assume that the parts $P_{1}, P_{2}, P_{3}$ are arranged in increasing order of size. (The ordered 3-ut property is a requirement on all ordered 3 -sets and all ordered 3-partitions, but having chosen the 3-set and 3-partition it suffices that the condition holds for all orderings of the 3 -set.) Using the transitivity of $G$, we may assume that $a_{1}$ can be mapped into $P_{1}$, and indeed that $a_{1} \in P_{1}$. Now the set

$$
\left\{(x, y):\left(a_{1}, x, y\right) \in\left(a_{1}, a_{2}, a_{3}\right)^{G}\right\}
$$

is the edge set of a Paley tournament on $\Omega \backslash\left\{a_{1}\right\}$. If this tournament includes an $\operatorname{arc}$ from $P_{2}$ to $P_{3}$, then we are done; so suppose not. If $\left|P_{2}\right|=1$, then $\left|P_{1}\right|=1$, and all arcs between $P_{2}$ and $P_{3}$ point into the point in $P_{2}$; so the tournament has out-degree at most 1 , a contradiction. So suppose that $\left|P_{2}\right|>1$. In the Paley tournament on $q$ points, any two points are dominated by precisely $(q-3) / 4$ points; but if there are no $\operatorname{arcs}$ from $P_{2}$ to $P_{3}$, then two points in $P_{2}$ are dominated by every point in $P_{3}$, and by assumption there are at least $q / 3$ such points. So $(q-3) / 4 \geq q / 3$, a contradiction.

So $\operatorname{PSL}(2, q)$, and any overgroup, has ordered 3 -ut for $q \equiv 3(\bmod 4)$.

We claim that, with the exceptions of $\mathrm{C}_{5}, \mathrm{D}(2 * 5)(n=5), \operatorname{AGL}(1,7)$ $(n=7), \mathrm{Sz}(8)$ and $\mathrm{Sz}(8): 3(n=65)$, types (b)-(e) in Proposition 2.7 are generously 2-transitive, and so have ordered 3-ut. For types (c),(d), and most groups of type (e), the 2-point stabilisers have all orbits of different sizes, these being 
- $2^{2 d-2} \pm 2^{d-1}-2$ and $2^{2 d-2}$ for type (c);

- $2^{2 d-1}-2$ and $2^{2 d-1}$ for type (d);

- 3, 6 for $\operatorname{PSL}(2,11)(n=11)$;

- 6,8 for $2^{4}: A_{6}(n=16)$;

- 6, 24 and 32 for $2^{6}: G_{2}(2)$ and its subgroup $(n=64)$;

- 12, 72 and 90 for HS $(n=176)$;

- 112 and 162 for $\mathrm{Co}_{3}(n=276)$.

For (b), since the point stabiliser has even order and rank at most 3, all its non-trivial orbitals are self-paired.

For the groups of type (f), if $q-1$ is not prime, then the point stabiliser of $\mathrm{A} \Gamma \mathrm{L}(1, q)(n=q)$ has a proper normal subgroup, and so these groups are not 2-primitive, and hence do not have ordered 3-ut. The same argument excludes $\operatorname{AGL}(1,5)(n=5)$ and $\operatorname{AGL}(1,7)(n=7)$.

Of the remaining groups, we observe that the Suzuki groups do not have ordered 3-ut since they are not 2-primitive (the point stabiliser has a normal subgroup of order $q$ ); and subgroups of index 2 in $\operatorname{AGL}(2, q)$ for odd $q$ fail to be 2-transitive, as do the 2 sporadic groups with $n=5$.

Hence the only open cases remaining are groups containing $\operatorname{AGL}(1, q)$ $(n=q)$ with prime $q-1$, which occurs only if $q=2^{p}$ for $p$ prime (and $2^{p}-1$ is a Mersenne prime).

Computation shows that $\operatorname{AGL}(1,8), \operatorname{A\Gamma L}(1,8)(n=8), \operatorname{AGL}(1,32)$ and $\mathrm{A \Gamma L}(1,32)(n=32)$ do indeed have ordered 3-ut.

\section{Strongly factorizable semigroups and maps with fixed rank}

A monoid $S$ with group of units $G$ and set of idempotents $E$ is said to be strongly factorizable if $S=E G$. Let $\Omega$ be a finite set. Every finite semigroup can be embedded in some $T(\Omega)$, a strongly factorizable semigroup. More generally, any semigroup $S$ such that $\operatorname{Sym}(\Omega) \leq S \leq T(\Omega)$ is strongly factorizable.

Let $k$ be a natural number; the goal of this section is to classify the groups $G \leq \operatorname{Sym}(\Omega)$ such that $\langle G, t\rangle$ is strongly factorizable for all rank $k$ 
transformation $t \in T(\Omega)$. The next result links this goal and the results of the previous sections.

Lemma 3.1 Let $\Omega$ be a finite set, $G \leq \operatorname{Sym}(\Omega)$ and $k \leq|\Omega|$. Then the following are equivalent:

(a) $G$ possesses the ordered $k$-ut property;

(b) for all rank $k$ transformations $t \in T(\Omega)$, we have

$$
\langle G, t\rangle=E G
$$

where $E$ is the set of idempotents of $\langle G, t\rangle$.

Proof First, assume that $G$ has the ordered $k$-ut property. Let $a \in\langle G, t\rangle$ be a map of rank $l \leq k$. As $G$ has ordered $k$-ut, it has ordered $l$-ut (Proposition $2.1(\mathrm{~b}))$. Therefore, given a sequence of kernel classes of $a$, say $\left(A_{1}, \ldots, A_{l}\right)$, and the corresponding $l$-tuple of images $\left(A_{1} a, \ldots, A_{l} a\right)$, there exists $g \in G$ such that $A_{i} a g \subseteq A_{i}$, for all $i \in\{1, \ldots, l\}$; thus $a g$ is an idempotent and $a=(a g) g^{-1} \in E G$. This proves the direct implication.

Conversely, let $\left(A_{1}, \ldots, A_{k}\right)$ be a $k$-partition of $\Omega$ and let $\left(a_{1}, \ldots, a_{k}\right)$ be a $k$-tuple of different elements of $\Omega$. We claim that there exists $g \in G$ such that $a_{i} g \in A_{i}$, for all $i \in\{1, \ldots, k\}$. In fact, let $t \in T(\Omega)$ be a map such that $A_{i} t=\left\{a_{i}\right\}$. By assumption $\langle G, t\rangle$ is strongly factorizable and hence $t=e g$, for some $g \in G$ and idempotent $e \in\langle G, t\rangle$, and thus $t g^{-1}=e$. Because $e$ and $t$ have the same kernel classes and every point in the image of $e$ is fixed, we have that $A_{i} e \subseteq A_{i}$ for all $i$. It follows that $\left\{a_{i} g^{-1}\right\}=A_{i} t g^{-1}=A_{i} e \subseteq A_{i}$ (for all $i \in\{1, \ldots, k\}$ ). The result follows.

Glueing together the previous result with the classification of the groups possessing the ordered $k$-ut in Propositions 2.4, 2.5, 2.6, and 2.8, we get Theorems 1.1-1.5 which are the main theorems of the first part of this paper.

\section{Semigroups and their normalizers}

Let $S \leq T_{n}$ be a semigroup and let $G \leq S_{n}$ be its normalizer in $S_{n}$. We are interested in the relation between $S$ and $\langle S, G\rangle$. On one hand the semigroup $\langle S, G\rangle$ might be more accessible to study since we can take advantage of 
group theoric results, but on the other hand the properties of $S$ might be very different from the properties of $\langle S, G\rangle$.

For example, we might be unable to verify if a given semigroup $S$ is regular. If all $t \in S$ have rank at most $k$ and if $G$ has the $k$-ut property, then the semigroup $\langle S, G\rangle$ is easily seen to be regular. Hence, to prove the regularity of $S$, we need to prove that regularity of $\langle S, G\rangle$ implies regularity of $S$. Therefore, the goal of this section is to study semigroup properties that carry from $\langle S, G\rangle$ to $S$.

We start by proving a general result.

Lemma 4.1 Let $S \leq T_{n}$ and let $G$ be its normalizer in $S_{n}$. Then

$$
\langle S, G\rangle=S G
$$

Proof For $s \in S$ and $g \in G$ let $s^{g}$ denote $g^{-1} s g$. Let $t \in\langle S, G\rangle$. We now have (for some $g_{1}, \ldots, g_{k+1} \in G, s_{1}, \ldots s_{k} \in S$ ),

$$
\begin{aligned}
t & =g_{1} s_{1} g_{2} s_{2} \ldots g_{k} s_{k} g_{k+1} \\
& =g_{1} s_{1} g_{1}^{-1}\left(g_{1} g_{2}\right) s_{2}\left(g_{1} g_{2}\right)^{-1}\left(g_{1} g_{2} g_{3}\right) \ldots\left(g_{1} \ldots g_{k}\right) s_{k}\left(g_{1} \ldots g_{k}\right)^{-1}\left(g_{1} \ldots g_{k} g_{k+1}\right) \\
& =s_{1}^{g_{1}} s_{2}^{g_{1} g_{2}} \ldots s_{k}^{g_{1} \ldots g_{k}}\left(g_{1} \ldots g_{k+1}\right) \\
& =s g \in S G,
\end{aligned}
$$

where $s=s_{1}^{g_{1}} s_{2}^{g_{1} g_{2}} \ldots s_{k}^{g_{1} \ldots g_{k}} \in S$ and $g=g_{1} \ldots g_{k+1} \in G$. Thus $\langle S, G\rangle \subseteq S G$. The reverse inclusion is obvious.

\subsection{Regularity}

Recall that a semigroup $S$ is regular if for all $a \in S$ there exists $a^{\prime} \in S$ such that $a=a a^{\prime} a$. Two elements $a, b \in S$ are said to be $\mathcal{R}$-related if there exist $u, v \in S^{1}$ such that $a=b u$ and $b=a v\left(S^{1}\right.$ denotes the monoid obtained by adjoining an identity to $S$ ). Similarly, $a, b \in S$ are said to be $\mathcal{L}$-related if there exist $u, v \in S^{1}$ such that $a=u b$ and $b=v a$. It is well know that a semigroup is regular if and only if every element is $\mathcal{R}$-related (or $\mathcal{L}$-related) to an idempotent. In what follows, by a transformation monoid $S \leq T_{n}$ we mean a semigroup of transformations containing the identity transformation.

The key result in this subsection is the following lemma.

Lemma 4.2 Let $S \leq T_{n}$ be a transformation monoid and $G$ be the normalizer of $S$ in the symmetric group. If $a \in S$ is $\mathcal{R}$-related in $S G$ to an idempotent of $S G$, then a is $\mathcal{R}$-related in $S$ to the same idempotent. 
Proof Let $a \in S$ and assume that $a$ is $\mathcal{R}$-related in $S G$ to an idempotent in $S G$, that is, there exist $b \in S, h \in G$ such that $(b h)(b h)=b h$ and for some $b_{1} i_{1}, b_{2} i_{2} \in S G$, we have $a=(b h)\left(b_{1} i_{1}\right)$ and $b h=a\left(b_{2} i_{2}\right)$. The claim is clearly true if $b h$ is the identity, so assume this is not case.

Now, by a theorem of McAlister, for all $s \in S$, the semigroups $\langle s, G\rangle \backslash G$ and $\left\langle g^{-1} s g \mid g \in G\right\rangle \backslash G$ have the same idempotents ( [24, Lemma 2.2] and [7, Lemma 2.2]). As $S=\bigcup_{s \in S}\left\langle g^{-1} s g \mid g \in G\right\rangle$ and $S G \backslash G=\bigcup_{s \in S}(\langle s, G\rangle \backslash G)$, it follows that $S$ and $S G$ have the same idempotents. Thus $b h \in S$.

It remains to prove that $a$ and $b h$ are $\mathcal{R}$-related in $S$, that is, there exist $u, v \in S$ such that $a=(b h) u$ and $b h=a v$. Since $(b h)\left(b_{1} i_{1}\right)=a \in S$, we can take $u=(b h)\left(b_{1} i_{1}\right)$ so that $a=(b h) u=(b h)(b h)\left(b_{1} i_{1}\right)$.

Observe that $a\left(b_{2} i_{2}\right)(b h)=(b h)(b h)=b h$. We claim that $i_{2} b h \in S$ and hence $b_{2} i_{2} b h \in S$, thus proving the theorem.

We start by proving that $h b h \in S$. In fact,

$$
\begin{aligned}
h^{-1} b h, b \in S & \Rightarrow h^{-1} b h b \in S \Rightarrow h^{-2} b h b h=h^{-2} b h \in S \Rightarrow h^{-2} b h b \in S \Rightarrow \\
& \Rightarrow h^{-3} b h b h=h^{-3} b h \in S \Rightarrow \ldots \Rightarrow h^{-k} b h \in S .
\end{aligned}
$$

As $G$ is finite, for some $k$ we have $h^{-k}=h$. The claim follows.

Now we claim that $i_{2}^{-k} b \in S$ for all natural numbers $k$. We proceed by induction. From $a, b_{2} \in S$ we get $a b_{2} \in S$ and hence $i_{2}^{-1} a b_{2} i_{2} \in S$, thus $i_{2}^{-1} b h \in S$ so that $i_{2}^{-1} b h b \in S$; as $b h b h=b h$, we have $b h b=b$ which together with $i_{2}^{-1} b h b \in S$ yields $i_{2}^{-1} b \in S$.

Now suppose that $i_{2}^{-k} b \in S$ (for some natural $k \geq 1$ ); we want to prove that $i_{2}^{-(k+1)} b \in S$. From $i_{2}^{-(k+1)} b_{2} i_{2}^{k+1}, i_{2}^{-k} b \in S$, we get $i_{2}^{-(k+1)} b_{2} i_{2}^{(k+1)} i_{2}^{-k} b=$ $i_{2}^{-(k+1)} b_{2} i_{2} b \in S$. Thus

$$
\begin{aligned}
S \ni\left(i_{2}^{-(k+1)} a i_{2}^{(k+1)}\right)\left(i_{2}^{-(k+1)} b_{2} i_{2} b\right) & =i_{2}^{-(k+1)} a i_{2}^{(k+1)} i_{2}^{-(k+1)} b_{2} i_{2} b \\
& =i_{2}^{-(k+1)} a b_{2} i_{2} b \\
& =i_{2}^{-(k+1)} b h b \\
& =i_{2}^{-(k+1)} b .
\end{aligned}
$$

It is proved that $i_{2}^{-k} b \in S$ for all natural $k$.

As $G$ is finite, for some $k$ we have $i_{2} b=i_{2}^{-k} b \in S$. Since $i_{2} b, h b h \in S$, it follows that $i_{2} b h b h=i_{2} b h \in S$. As $b_{2}, i_{2} b h \in S$, we get $b_{2} i_{2} b h \in S$ and hence $a\left(b_{2} i_{2} b h\right)=\left(a b_{2} i_{2}\right)(b h)=(b h)^{2}=b h$. It is proved that $a$ and the idempotent bh are $\mathcal{R}$-related in $S$. 
By symmetry we get the following.

Lemma 4.3 Let $S \leq T_{n}$ be a transformation monoid and $G$ be the normalizer of $S$ in the symmetric group $G$. If $a \in S$ is $\mathcal{L}$-related in $S G$ to an idempotent of $S G$, then a is $\mathcal{L}$-related in $S$ to the same idempotent.

Two elements $a, b \in S$ are said to be $\mathcal{H}$-related if they are $\mathcal{R}$-related and $\mathcal{L}$-related. The two previous results imply the following.

Lemma 4.4 Let $S \leq T_{n}$ be a transformation monoid and $G$ be the normalizer of $S$ in the symmetric group $G$. If $a \in S$ is $\mathcal{H}$-related in $S G$ to an idempotent of $S G$, then a is $\mathcal{H}$-related in $S$ to the same idempotent.

A number of consequences follow from these lemmas.

Corollary 4.5 Let $S \leq T_{n}$ be a transformation monoid and $G$ be the normalizer of $S$ in the symmetric group $S_{n}$. Then $S G$ is regular if and only if $S$ is regular.

Proof Let $a \in S$. As $S G$ is regular and $S \leq S G$, it follows that $a$ is $\mathcal{R}$ related in $S G$ to an idempotent of $S G$. By Lemma 4.2, $a$ is $\mathcal{R}$-related in $S$ to the same idempotent (which is thus in $S$ ). We conclude that every element in $S$ is $\mathcal{R}$-related to an idempotent.

Regarding the converse, suppose $S$ is regular, say $s=s s^{\prime} s$ and $s^{\prime}=s^{\prime} s s^{\prime}$, for all $s \in S$. Let $s g \in S G$. Then $s g=(s g)\left(g^{-1} s^{\prime}\right)(s g)$ and $g^{-1} s^{\prime}=$ $\left(g^{-1} s^{\prime}\right)(s g)\left(g^{-1} s^{\prime}\right)$; in addition $g^{-1} s^{\prime}=\left(g^{-1} s^{\prime} g\right) g^{-1} \in S G$. It is proved that every element in $S G$ has an inverse in $S G$. The result follows.

We observe that it is possible for non-idempotents $p, q \in S$ to be $\mathcal{R}$-related in $S G$, but not in $S$. For example, pick $g, t, q \in T_{7}$ as follows: $g:=(567)$,

$$
t:=\left(\begin{array}{cccc}
1,2,3,4 & 5 & 6 & 7 \\
1 & 2 & 3 & 4
\end{array}\right) \quad \text { and } \quad q:=\left(\begin{array}{cccc}
1,2,3,4 & 5 & 6 & 7 \\
1 & 3 & 4 & 2
\end{array}\right)
$$

Then $S:=\langle g, t, q\rangle$ has 7 elements and its normalizer $G$ in $S_{7}$ is generated by $\langle g,(34)(76),(23)(76)\rangle$. We have $t=q(243)$ and $q=t(234)$, thus $t$ and $q$ are $\mathcal{R}$-related in $S G$, but they are not $\mathcal{R}$-related in $S$.

Recall that a semigroup is completely regular if each element belongs to a maximal subgroup; equivalently, every $\mathcal{H}$-class contains an idempotent. Therefore, Lemma 4.4 implies the following. 
Corollary 4.6 Let $S \leq T_{n}$ be a transformation monoid and $G$ be the normalizer of $S$ in the symmetric group $S_{n}$. Then $S G$ is completely regular if and only if $S$ is completely regular.

An element $a$ of a semigroup $S$ is said to be abundant if it is $\mathcal{R}$-related and $\mathcal{L}$-related to an idempotent in some oversemigroup $T$ of $S$. Therefore, if an element $a \in S$ is abundant in $S G$, this means that $a$ is also abundant in $S$. The abundant world generalizes the regular world, but in this context adds nothing.

An element $a$ of a semigroup $S$ is said to be right [left] inverse if it is $\mathcal{R}$-related $[\mathcal{L}$-related] to exactly one idempotent in $S$; the element is inverse if it is both left and right inverse. A semigroup is inverse if all of its elements are inverse. As seen above, if $a \in S$ is $\mathcal{R}$-related to exactly one idempotent in $S G$, by Lemma 4.2 we know that in $S$ the element $a$ is $\mathcal{R}$-related to the same idempotent (that belongs to $S$ ); thus, if $a \in S$ is right inverse in $S G$, it is also right inverse in $S$; as a consequence, if $S G$ is an inverse semigroup, then so is $S$. (This last conclusion follows immediately from the fact if $S G$ is inverse, it is regular and the idempotents commute and hence the same is true in $S$.)

A semigroup is said to be Clifford if it is inverse and completely regular. By the results above it follows that if $S G$ is Clifford, then so is $S$.

A monoid $S$ is intra-regular if for all $a \in S$ there exist $b, c \in S$ such that $b a^{2} c=a$. We would like to know if $S G$ intra-regular implies $S$ intra-regular, but we only have the following partial result.

Proposition 4.7 Let $G \leq S_{n}$ be a group of exponent 2 and let $S \leq T_{n}$ be a transformation monoid such that $G$ is the normalizer in $S_{n}$ of $S$. Then $S G$ is intra-regular implies that $S$ is intra-regular.

Proof As $S$ is a finite semigroup, for each $x \in S$ there exist natural numbers $l$ and $m, m>l$, such that $x^{l}=x^{m}$.

Let $a \in S$ be arbitrary, and $l$ and $m$ as above. If $l=1$ and $m=2$, then $a=a^{2}$ and hence $a=1 a a 1$, with $1 \in S$; if $l=1$ and $m>2$, then $a=1 a^{2} a^{m-2}$, so the result holds for all $a$ for which $l=1$.

Assume instead that $l, m>1$. By intra-regularity in $S G$, there exist $e g, f h \in S G$ such that $a=e g a^{2} f h$, with $e, f \in S$ and $g, h \in G$. It is clear that $f, h f h^{-1} \in S$ and hence $f h f h^{-1} \in S$; as $h^{-1}=h$ it follows that 
$f h f h \in S$. We claim that egaeg $\in S$. In fact ae $\in S$ because $a, e \in S$; thus gaeg $^{-1}, e \in S$ and hence $e g a e g^{-1}=e g a e g \in S$. The claim follows. Now

$$
(e g a e g) a^{2}(f h f h)=e g a\left(e g a^{2} f h\right) f h=e g a(a) f h=e g a^{2} f h=a .
$$

It is proved that every intraregular element in $S G$ is intraregular in $S$, when $G$ satisfies $x^{2}=1$.

\subsection{Semigroups having $n$-th roots}

The aim of this subsection is to carry the foregoing investigation to the case of $S G$ having $n$-th roots. Observe that every finite semigroup satisfies an identity of the form $x^{m}=x^{k}$, for $0 \leq k<m$. If $a, b \in S$ and $b^{n}=a$ we say that $b$ is an $n$-th root of $a$; we denote an arbitrary $n$th root of $x$ by $\sqrt[n]{x}$ and the notation $\sqrt[n]{x} \in A \subseteq S$ means that $x$ has an $n$-th root in the set $A$.

Theorem 4.8 Let $S$ be a finite semigroup in which every element has an $n$-th root, for some $n \geq 2$. Then for every $x \in S$ we have $\sqrt[n]{x} \in\langle x\rangle$.

Proof Let $s: S \rightarrow S$ given by $s(x)=x^{n}$. That $S$ has $n$-th roots for all of its elements means that $s$ is surjective, and as $S$ is finite, it is also injective. But for any $x \in S, s(\langle x\rangle) \subseteq\langle x\rangle$. Let $t$ be the restriction of $s$ to $\langle x\rangle$. Then $t$ is injective (because $s$ is), and by finiteness, also surjective. Hence $x$ has a (unique) $n$-th root in $\langle x\rangle$.

Now back to the leitmotiv of this paper.

Corollary 4.9 Let $S \leq T_{n}$ be a transformation monoid and $G$ be the normalizer of $S$ in the symmetric group $S_{n}$. If every element in $S G$ has a $k$-th root, then so has every element in $S$.

Proof As $S G$ has $k$-th roots for all its elements, it follows that for every $x \in S G$ we have $\sqrt[k]{x} \in\langle x\rangle$ and hence every $x \in S$ has a $k$-th root in $S$.

Let $n$ be a positive integer. Then every element of the finite group $G$ has an $n$-th root if and only if $n$ is coprime to the order of $G$. For, if $n$ and $|G|$ have a common factor $p$, and $g \in G$ has order $p$, then $g^{n}=1^{n}$, so the $n$-th power map is not injective; conversely, if $\operatorname{gcd}(n,|G|)=1$, choose $m$ so that $m n \equiv 1(\bmod |G|)$; then the $n$-th and $m$-th power maps are mutually inverse on $G$. 
Corollary 4.10 Let $n$ be a positive integer. Then every element of the finite semigroup $S$ has an $n$-th root in $S$ if and only if every element of $S$ is contained in a maximal subgroup of $S$ of order coprime to $n$.

Proof Let every element of $S$ have an $n$th root, and take $x \in S$. Then, by Theorem 4.8, $\sqrt[n]{x} \in\langle x\rangle$, that is, $\sqrt[n]{x}=x^{k}$ for some natural number $k$. This implies that $x=x^{n k}$, so that $x^{n k-1}$ is an idempotent which is the identity of the cyclic group generated by $x$. This means that the $\mathcal{H}$-class of $x$ is a group. In this group, every element has an $n$-th root, so the order of the group is coprime to $n$.

Conversely, if $S$ is a union of groups of orders coprime to $n$, then every element of $S$ has an $n$-th root.

It is well known that, for example, neither the symmetric group nor the full transformation monoid contain square roots of all their elements, except in trivial cases. With our main theorem at hand, we can say a bit more. If $S$ is a finite semigroup and some element $a$ has no $n$-th root, then it is not possible to extend $S$ to a finite semigroup $T$ containing an $n$-th root for all elements (because an $n$-th root of $a$ must belong to $\langle a\rangle$, and this semigroup remains the same in any oversemigroup of $S$ ).

\subsection{A negative result}

A semigroup $S$ is said to be $\mathcal{R}$-commutative if for all $a, b \in S$ we have $a b \mathcal{R} b a$. Let $S<T(\{1, \ldots, 7\})$ be the semigroup generated by the permutations (24)(36), (15)(23)(46) and the transformation

$$
t=\left(\begin{array}{ccc}
\{1,5,7\} & \{2,3\} & \{4,6\} \\
7 & 1 & 5
\end{array}\right) .
$$

The normalizer $G$ of $S$ in the symmetric group is the group generated by (15), (24)(36) and (15)(23)(46). GAP shows that the semigroup $S G$ is $\mathcal{R}$ commutative, but $S$ is not. By symmetry, a corresponding counterexample exists for $\mathcal{L}$-commutativity.

\section{Problems}

We now propose a number of problems. The first is essentially in [5] but (annoyingly) keeps resisting. 
Problem 1 Complete the classification of the groups possessing the 3 - and 4-ut property so that Theorem 1.4 and Theorem 1.5 can be completed.

There is a well known correspondence between the behaviour of $T(\Omega)$ and $\operatorname{End}(V)$, when $\Omega$ is a finite set and $V$ is a finite dimension vector space. This prompted the introduction of independence algebras, a class containing both sets and vector spaces as particular cases. Therefore the next two problems turn out to be very natural.

Problem 2 Prove linear analogues of the main theorems in this paper.

Problem 3 Find in the context of independence algebras analogues of the main theorems in this paper.

Let $S$ be a finite semigroup. Two elements $a, b \in S$ are said to be $\mathcal{J}$ related if they generate the same principal ideal, that is, $S^{1} a S^{1}=S^{1} b S^{1}$. We recall ( $[17$, p.57]) that if $S<T$ is a regular subsemigroup of a semigroup $T$, then $\mathcal{R}_{S}=\mathcal{R}_{T} \cap(S \times S)$. The same happens for $\mathcal{L}$ or $\mathcal{H}$, but fails for $\mathcal{J}$.

Problem 4 Let $S \leq T_{n}$ and $G \leq S_{n}$ be its normalizer in $S_{n}$. Is it true that $\mathcal{J}_{S}=\mathcal{J}_{S G} \cap(S \times S) ?$

An existential property of semigroups is a first order language condition on the elements of the semigroup that uses an existential quantifier. For example, regularity is an existential property of semigroups.

Problem 5 Let $S \leq T_{n}$ be a semigroup and $G$ its normalizer in $S_{n}$. Let $P$ be an existential property of semigroups. Decide if $S G$ satisfies $P$ implies that $S$ also satisfies $P$.

The following result was proved for groups satisfying $g^{2}=1$. Can it be generalized for other classes of groups?

Problem 6 Let $G \leq S_{n}$ be a group and let $S \leq T_{n}$ be a transformation monoid such that $G$ is its normalizer in $S_{n}$. Is it true that if $S G$ is intraregular then $S$ is intra-regular?

We close the list of problems with a general semigroup structure question.

Problem 7 Consider $G$, one of the groups appearing in Theorems 1.1-1.5. Describe the structure (Green's relations, automorphisms, congruences, conjugacy classes, the variety generated, etc.) of the semigroups $\langle G, a\rangle$, for $a \in T_{n}$. 


\section{Acknowledgements}

We thank the referee for an excellent report that led to an improved version of this paper.

The first author was partially supported by the Fundação para a Ciência e a Tecnologia (Portuguese Foundation for Science and Technology) through the projects UIDB/00297/2020 (Centro de Matemtica e Aplicaes), PTDC/MATPUR/31174/2017, UIDB/04621/2020 and UIDP/04621/2020.

\section{References}

[1] J. André, J. Araújo and P.J. Cameron. The classification of partition homogeneous groups with applications to semigroup theory. Journal of Algebra, 452, (2016), 288-310.

[2] J. Araújo, W. Bentz and P. J. Cameron, Groups synchronizing a transformation of non-uniform kernel. Theoret. Comput. Sci., 498 (2013), $1-9$.

[3] J. Araújo, W. Bentz and P. J. Cameron, Orbits of primitive $k$ homogenous groups on $(n-k)$-partitions with applications to semigroups, Transactions of the American Mathematical Society 371 (2019), $105-136$.

[4] J. Araújo, W. Bentz and P. J. Cameron, The existential transversal property: a generalization of homogeneity and its impact on semigroups, submitted. https://arxiv.org/abs/1808.06085

[5] J. Araújo and P. J. Cameron, Two generalizations of homogeneity in groups with applications to regular semigroups, Trans. Amer. Math. Soc. 368 (2016), 1159-1188.

[6] J. Araújo, W. Bentz, P.J. Cameron, G. Royle and A. Schaefer, Primitive groups, graph endomorphisms and synchronization. Proceedings of the London Mathematical Society 113 (2016), 829-867.

[7] J. Araújo, J.D. Mitchell and C. Schneider. Groups that together with any transformation generate regular semigroups or idempotent generated semigroups. Journal of Algebra, 343, 1 (2011), 93-106. 
[8] J. Araújo and P. J. Cameron, Primitive groups synchronize non-uniform maps of extreme ranks, Journal of Combinatorial Theory, Series B, 106 (2014), 98-114.

[9] J. Araújo, P.J. Cameron, J.D. Mitchell and M. Neunhöffer, The classification of normalizing groups. Journal of Algebra 373 (2013), 1: 481 490.

[10] J. Araújo, P.J. Cameron and B. Steinberg, Between primitive and 2transitive: Synchronization and its friends. EMS Surveys in Mathematical Sciences 4:2 (2017), 101-184.

[11] F. Catino, Factorizable semigroups, Semigroup Forum 36 (1987), 167174 .

[12] D. G. FitzGerald, Factorizable inverse monoids, Semigroup Forum, 80, (2009), 484-509.

[13] Fredrick Arnold and Benjamin Steinberg. Synchronizing groups and automata. Theoret. Comput. Sci. 359 (1-3) (2006),101-110.

[14] Peter J. Cameron and Priscila A. Kazanidis. Cores of symmetric graphs. J. Aust. Math. Soc. 85 (2) (2008), 145-154.

[15] The GAP Group. GAP - Groups, Algorithms, and Programming, Version 4.9.1, http://www . gap-system.org/

[16] J. east, Idempotents and one-sided units in infinite partial Brauer monoids, J. Algebra 543 (2019), 427-482.

[17] John M. Howie. Fundamentals of semigroup theory, volume 12 of London Mathematical Society Monographs. New Series. The Clarendon Press Oxford University Press, New York, 1995. Oxford Science Publications.

[18] William M. Kantor. k-homogeneous groups. Math. Z. 124 (1972), 261-265.

[19] I. Levi, D. B. McAlister, and R. B. McFadden. Groups associated with finite transformation semigroups. Semigroup Forum, 61 (3) (2000), 453467. 
[20] I. Levi and R. B. McFadden. $S_{n}$-normal semigroups. Proc. Edinburgh Math. Soc. (2), 37 (3) (1994), 471-476.

[21] I. Levi. On the inner automorphisms of finite transformation semigroups. Proc. Edinburgh Math. Soc. (2), 39 (1) (1996), 27-30.

[22] M. W. Liebeck, C. E. Praeger and J. Saxl, On factorizations of almost simple groups, J. Algebra 185 (1996), 409-419.

[23] M. W. Liebeck, C. E. Praeger and J. Saxl, Transitive subgroups of primitive permutation groups, Journal of Algebra 234 (2000), 291-361.

[24] Donald B. McAlister. Semigroups generated by a group and an idempotent. Comm. Algebra, 26 (2) (1998), 515-547.

[25] Peter M. Neumann. Primitive permutation groups and their sectionregular partitions. Michigan Math. J., 58 (2009), 309-322.

[26] K. Tolo, Factorizable Semigroups, Pacific Journal of Mathematics 31 (2) (1969), 523-535. 